\title{
Evaluation for the Anthropocene: Challenges ahead and making it happen
}

\section{E. Jane Davidson}

Fellows' Strand plenary

Evaluation for the Anthropocene: Shaping a sustainability-ready evaluation field

2018 Canadian Evaluation Society Conference

My evaluator friends, if you find your heads spinning with the fascinating deep insights of these sustainability gurus, if you are wondering how to conceptually weave their insights and draw out the practical implications for us as evaluators, you are in good company!

I am no sustainability expert myself. I came to this panel discussion bringing a background in the so-called "hard" sciences as well as (more deeply) in organisational psychology. I have long had an interest in organisational and systems change, including how we change thinking and behaviours and whole systems, so I have used that lens as a way of thinking about the contributions of the other panellists.

My first thought was this. How did we, as evaluators, get stuck in this space where we are primarily focused on the "human systems" side of things but tend to ignore the "natural systems"? 
Why do we gravitate towards thinking within the boundaries of the programme, the policy, or the initiative, and what its intended outcomes were-at the expense of thinking outside that frame?

There are some practical reasons, for sure, but from what we have heard from the panel, I think the big one is subconscious for most of us. It's the pervasiveness of the Western and colonial worldview that drives policy, programming, and development, and seeps through from the perspectives we are fed daily by the mainstream media. It's about "man" [sic] being the Supreme Being in charge of all the various resources on Planet Earth.

In this worldview, there's an emphasis on entitlement rather than responsibility and stewardship. Earth's resources are simply there to serve humans and to be plundered. As Sean said, the environment, environmental regulations, conservationists- "those greenies" as they call us where I come from-are just annoyances that you've got to get past as you get on with the most important stuff, which is chasing business and economic growth.

This worldview is deeply entrenched, and not just in people's thinking and reasoning. It's also deeply embedded in people's very identities - their political identities and their cultural identities. This is what makes it so hard to shift.

Let me explain.

Each of us has an identity that is partly individual (our unique personality) and partly a blend of the various different groups with which we identify and who influence our thinking. We have elements of our identity that derive from being of a particular political persuasion, or being part of an industry, or hailing from a particular academic discipline, and from the various ethnicities and subcultures with which we identify, for example.

Each of these group identity elements has a "culture" associated with it, which includes a particular way of thinking, talking, and 
making sense. Economists think about the world differently from psychologists, and so on. These ways of making sense are reflective of a set of underlying beliefs, values, and assumptions about the nature of the world and the place of humans within it.

When assumptions like "the world's resources exist to be plundered for economic gain" are not just consciously held beliefs, but deepseated worldviews strongly associated with one's very identity, they are significantly harder to shift. Unearthing and challenging those assumptions may be seen as a personal attack on those who hold them.

On top of that challenge, we have the psychology and politics of accountability for results. People often react to programme and policy evaluation as if it were an appraisal of their performance as individuals. As a natural extension, people prefer that the programmes and policies with which they are involved are only evaluated on criteria over which they have complete control. When we start trying to include sustainability-related outcomes in evaluation, there's a lot of pushback on the grounds that these are affected by many other influences and actors, so it is "unfair" to hold the programme or policy accountable for them.

To be blunt, that's a planet-killing cop-out as well as a push for an "outputs only; no outcomes" approach. It'd be like teachers saying they can only be held responsible for the quality of their teaching and not whether their students actually learn anything from it.

There's a related issue in play as well, around where an evaluation's boundaries are drawn. For evaluations that are commissioned by government agencies, the very nature of these entities means that their focus is almost always within national borders and on the things that could affect citizens and residents, not those living in the country next door.

This is in sharp contrast to a Blue Marble Evaluation view, where there's an explicit recognition that side effects and side 
impacts - particularly impacts on natural systems — show a complete disregard for these artificial lines humans draw on maps (Patton, 2019). Although government agencies may be acting locally, the impacts of their work are often global.

As we zoom back in within the boundaries, there are many more forces that work to keep the evaluation inside the "human systems" box and away from "natural system" impacts.

Evaluation funders tend to want to keep the evaluation tightly contained, often for budget and timeline reasons, but just as often because they don't want weaknesses exposed on things they hadn't really been thinking about or taking action on. As evaluators, we tend to play along with this in order to win the contract, and it is often exceedingly difficult to negotiate a broadening of scope once the work is under way.

Even if we did manage to broaden the scope of evaluation to get sustainability more front and central, the truth is that most of us are a little short on the expertise that's needed to do solid sustainability-ready evaluation. I can't recall seeing this topic in too many graduate evaluation courses, can you?

Given all these challenges, what needs to happen if the evaluation enterprise is to make a shift towards more coupled systems-focused and sustainability-ready evaluation?

I think the first one is what Andy said at the beginning about having one of our guiding core values for all of our evaluation work as this: Whatever we're evaluating needs to be meeting and supporting the needs and aspirations of the communities but without compromising in any way the ability of future generations to enjoy and thrive in the natural environment.

When I think about what we need in order to start doing that, it's not just about shifting our beliefs and attitudes but also thinking about what we need to do to lift our knowledge and evaluative know-how. 
A lot of the methods and tools coming out of this space are very quantitative. They're very dollar-oriented as well, and as a result they don't do a great job of capturing some stuff that's really important but quite intangible and a little bit difficult to measure-things like the cultural significance of certain environmental treasures or thinking about sustainability and outcomes in multiple generation terms. A potentially fruitful emerging methodology is Julian King's work on Value for Investment (VfI), which combines economics methods with rubrics methodology in ways that can help answer the VfI question even when some of the important considerations are intangible or unquantifiable. ${ }^{1}$

Another thing we need to bring to the table as evaluators is pushing people to include human and natural systems-related questions in the list of high-level key evaluation questions that should guide every evaluation. That means not stopping at asking "What were the outcomes?" or even "How good were the outcomes?" I am talking about asking a higher-level question: "How well is this initiative trading off the various benefits and drawbacks across those two coupled systems?"

Answering big-picture evaluative questions like that is going to require consensus building and involvement of multiple stakeholders with a wide range of expertise. It's going to require that broader theory of change that was mentioned earlier in the panel. But it's also going to require some new frameworks and tools to get the job done.

Too often in evaluation, there's a tendency to let the methods drive the evaluation questions. That's because, when most of us were trained in applied social science research methods, we were taught to make sure that any research questions could be operationalised, and that objectives should be SMART (specific, measurable, etc.). It's not just the evaluators, either. How many times have you asked your stakeholders what questions they want answered, only to get a response along the

1 For a quick summary and links to the latest resources, see https://www.julianking.co.nz/vfi/ 
lines of "We want to know how much [indicator X] has shifted." They are limiting their questions to what they know can be measured, rather than thinking of the broader (but more difficult) questions they actually need answered in order to feed into their thinking or decision making.

When we can get stakeholders to let go of their "but how are we going to measure it?" worries, we can guide them towards identifying the evaluative questions they truly need answered, and to let us worry about how we are going to make that happen. After all, we evaluators dream about that stuff every night, right?

OK, so how do we move our discipline forward on sustainability-ready evaluation? Here, I want to bring in another little gem from the organisational change literature and ask you all a question.

Which category are you in? And what about the colleagues around you?

Are you one of the innovators like these sustainability gurus up here on the panel? Are you thinking, "Yeah, let's gather up the ideas we have already, and invent some new stuff to fill the gaps, so that we can get out there and do this!"

Are you an early adopter-someone who's thinking "That sounds like a great idea ... Now can you just tell me how to do it?"

Are you in the wait-and-see early majority? "I think this might be a good idea, but I just want to see how it works out, see a few points on the board before I jump in ...”

Then you've got the late majority folks who are a little bit wary about this and they're more looking for political rather than rational reasons to shift. You know the type; you've seen them at work when there's a change coming down the line, thinking "Hmmmm, I'm not that keen on this so am not about to jump in, but if it starts taking off, at what point do I start looking like a stick-in-the-mud change resister when everyone else is getting on board? I'll just bide my time until it looks like I'm going to have to join the shift." 
Further down the chain we have the resisters. They are apathetic about the whole idea and pretty much just try to duck and ignore it. "Ugh, not another change that's going to require pointless effort. Let's just hope it goes away because we all know the next thing will come along soon enough and then we won't have to worry about it."

Finally, the ones you've really got to keep an eye on are the saboteurs. They are the ones who will work actively or passively to undermine the whole idea because they're fundamentally against it and/or dislike whoever is leading the change.

Resisters and saboteurs are highly likely to be the people for whom plundering natural resources and ignoring the sustainability issue is an important but undiscussable part of their cultural and political identity. As a result, they will be hardest to shift, and it will take a whole lot more than evidence and rational reasoning. I won't go into huge detail here, but briefly, the knowledge base from organisational change suggests that the only options for getting these folks to change are shifting the system and the culture around them and making the tide of change so strong that it will be impossible to swim against.

The early and late majorities (both evaluators and stakeholders) are going to need some good workable exemplars and clear evidence that sustainability-focused evaluation is being positively received and is well on the way to becoming the wave of the future. They will also need reassurance that the focus is on critiquing not them personally but "the [human] system" and how effectively it is coupled with the natural system. In other words, evaluation will help identify the constraints that prevent them from being able to function more sustainably rather than blaming them personally for failures.

But what about those of us wanting to help lead the change? If you're an innovator with some ideas ready to go, or an early adopter looking for something you can get your teeth into, let's think about what we can do and whose brains we need to pick to get this show on the road. 
An obvious first move is to spend some time with these sustainability gurus - read what they've written already, tune into their sessions at conferences, write into your next contract enough budget so you can pick their brains for a few hours and pool your expertise with theirs to make sure you are on the right track.

There's another significant and growing group of evaluators whose expertise is pure gold here, and that is the flourishing community of indigenous evaluators around the world. When you have grown up knowing that you belong to the land (not the other way around) and with a multi-generational perspective on sustainability, that puts you in a strong position to effectively weave evaluation theory and methodology with this sustainability-infused worldview that is already part of who you are. The importance of indigenous expertise was recently recognised with unprecedented clarity in a major report from the United Nations Environment Programme (2019). ${ }^{2}$ The evaluation community is gradually waking up to the realisation that indigenous evaluation theory has a whole lot more to offer than simply expertise to evaluate "cultural" programmes. Set your alarms a bit louder, folks - we need to wake the rest up!

Now, one thing I want to say is that this may look like it's going to be hard work to push and get people thinking differently. It's not just evaluators; it's clients; and it's those big influential organisations that set the agenda for all kinds of evaluation around the worldespecially in sectors like International Development where you've got several very big heavy hitters setting agendas for everybody else.

I want to remind us that we've actually been here before as evaluators and we've done some of this already on multiple different fronts. Don't believe me? Let me jog your memory (or, share a bit of history if you are new to the profession).

2 https://www.unenvironment.org/news-and-stories/press-release/natures-dangerous-decline-unprecedented-species-extinction-rates 
Once upon a time evaluation was defined as "measuring whether a program met its objectives." Remember when we talked to clients about how side effects and side impacts were important too? Naturally, they pushed back. They initially only wanted to be evaluated on what they had intended to achieve, not a bunch of stuff that happened accidentally. But then we pointed out that, from the perspective of impactees, their families and communities, it didn't much matter whether something was intended or not; what mattered was whether it happened and how important it was. And so, eventually, they agreed that we should look at all important outcomes, intended or not.

Looking at sustainability and the impact of human systems on natural systems is simply an extension of what we do when we look for unintended effects, both positive and negative. It's just another frame for doing so.

Once upon another time, we had a lot of clients with a strong preference for quantitative methods or for particular kinds of metrics. Gradually we helped them understand that quantitative evidence makes for nice "bones," but every good piece of research puts some qualitative "flesh" on those bones, and every good evaluation brings them both to life with the "beating heart" that is evaluative reasoning (Davidson, 2014). It was (and still is) a long process to get people on board with using mixed methods, and then another long process again to get them to understand the value of actually evaluative evaluation. Over time, we gradually get headway. We've just got to keep bashing on those doors and convincing people.

I want to encourage us to identify the key influencers that we each have access to. Not just evaluators, but others out there in politics, policy, and the media. If we can find those who have a platform and push their thinking a little bit more, if we all keep chipping away at it, we'll get somewhere eventually_like we have in the past. 
In closing, I want to leave you with this.

In the same way as culturally responsive evaluation is not something that we should just apply to those "cultural" programmes, sustainability-ready evaluation is not just something we should just apply to those "environmental" programmes. In both cases, it's the responsibility of all of us to hold policy and programming accountable for being both culturally responsive and environmentally sustainable.

Ultimately, it's about our relationship with Mother Earth. We don't own her; she owns us. If we screw this up, guess what-Mother Earth is the most exacting meta-evaluator you are ever going to have to deal with!

Kia ora.

\section{References}

Davidson, E. J. (2014). How "beauty" can bring truth and justice to life. In J. C. Griffith \& B. Montrosse-Moorhead (Eds.), Revisiting truth, beauty, and justice: Evaluating with validity in the 21st century. New Directions for Evaluation, 142, 31-43.

Patton, M. Q. (2019). Blue marble evaluation: Premises and principles. New York: Guilford.

\section{The author}

Dr Jane Davidson is an internationally recognised evaluation thought leader, best known for developing evaluation rubrics as a methodology for drawing conclusions about quality and value. See http:// realevaluation.com/about/jane/

Email: realevaluation@gmail.com 